\title{
Electromagnetic effects and the longitudinal evolution of the system at CERN SPS energies
}

\author{
N. Davis*, A. Rybicki, A. Szczurek, I. Sputowska, A. Marcinek, V. Ozvenchuk, \\ M. Kiełbowicz and S. Bhosale \\ Institute of Nuclear Physics, Polish Academy of Sciences \\ E-mail: nikolaos.daviseifj.edu.pl
}

\begin{abstract}
We review our studies of spectator-induced electromagnetic (EM) effects on charged pion emission in nucleus-nucleus collisions at CERN SPS and RHIC BES energies. These we discuss in the context of (1) new data on $\mathrm{Ar}+\mathrm{Sc}$ collisions from the NA61/SHINE experiment (2) new findings on the role of energy-momentum conservation for the longitudinal evolution of the system at SPS energies, and (3) new work on the space-time evolution of spectator fragmentation. Although the average Ar spectator charge in intermediate $\mathrm{Ar}+\mathrm{Sc}$ collisions is only about 8 elementary units, the corresponding EM field is large enough to impose a visible distortion on final state $\pi^{+} / \pi^{-}$ratios, and break isospin symmetry [1]. A Monte Carlo simulation of this process provides new information on the space-time evolution of the system in Ar+Sc collisions, as well as that of spectator fragmentation. We compare this information to that obtained for $\mathrm{Au}+\mathrm{Au}$ and $\mathrm{Pb}+\mathrm{Pb}$ collisions from STAR [2], NA49 [3], and WA98 [4] experiments. A uniform picture emerges where the distance $d_{E}$ between the pion formation zone at freeze-out and the spectator system decreases with increasing pion rapidity. At central rapidity our estimates agree with pion decoupling times obtained from standard femtoscopy [5]. As a result, a specific picture of the longitudinal evolution of the system emerges. We construct a simple model of the heavy ion collision, local in the impact parameter plane, and appropriate for the SPS energy range. With some similarity to the original "fire-streak" approach, we start from local energy and momentum conservation, and nicely describe the centrality dependence of the pion rapidity distribution and total pion yields in heavy ion collisions at $\sqrt{s_{N N}}=17.3 \mathrm{GeV}$ [6]. We also explain the broadening of this distribution when going from central to peripheral collisions. We discuss the resulting implications on the role of energy and momentum conservation in the early stage of the A+A reaction [7]. Finally, we comment on the possibility of using EM effects in relativistic heavy ion collisions to test the nuclear models of spectator break-up [8]. This includes possible new measurements in the framework of the NA61/SHINE Phase II programme recommended by the SPSC [9].
\end{abstract}

Corfu Summer Institute 2018 "School and Workshops on Elementary Particle Physics and Gravity" (CORFU2018)

31 August - 28 September, 2018

Corfu, Greece

${ }^{*}$ Speaker. 


\section{Introduction}

In a heavy ion collision experiment, we can distinguish between two different sub-systems that play distinct roles in the evolution of the system: on the one hand, there is the participant zone, formed by the nucleons which take part in the collision and produce the resulting fireball; on the other hand, there is the system of spectators, nuclear remnants that fly away from the collision point at approximately beam rapidity. In non-central collisions, spectators carry significant charge and therefore generate strong electromagnetic fields. The latter modify the trajectories of final state charged particles produced in the collision. Positively (negatively) charged particles are repelled by (attracted towards) the spectator system, which results in charge asymmetries induced in the distributions of charged particles.

Recent studies indicate that spectator-induced electromagnetic (EM) effects are sensitive to the space-time evolution of the system, and can therefore be used to provide information on the subject. In the present work, we review a number of observables and models related to spectator-induced EM effects on charged pion emission in nucleus-nucleus collisions at CERN SPS and RHIC BES energies.

\section{Electromagnetic effects \& charge asymmetries}

\subsection{EM distortion of $\pi^{+} / \pi^{-}$ratio}

One of the main observables where we expect to see spectator-induced EM effects is the finalstate distribution of pions produced in a peripheral nucleus-nucleus collision, and specifically in the ratio of positively over negatively charged pions, $\pi^{+} / \pi^{-}$.

Fig.1(a) shows the ratios $\left(\pi^{+} / \pi^{-}\right)$, at several values of $p_{T}$, of peripheral $P b+P b$ collisions by the fixed target NA49 experiment at CERN SPS [10] plotted as a function of Feynman variable $x_{F}$,

$$
x_{F} \equiv \frac{p_{L}}{p_{\text {beam }}},
$$

where $p_{L}$ is the pion longitudinal momentum and $p_{\text {beam }}$ is the beam longitudinal momentum in the collision c.m.s. We observe an excess of negative over positive pions for the whole kinematic range; for higher values of transverse momentum, the slight excess present can be explained by isospin effects resulting from the composition of the projectile $\mathrm{Pb}$ nucleus (surplus of neutrons over protons in the nucleus). For low $p_{T}$ values, however, we observe a characteristic isospin-symmetry breaking structure, consisting of a sharp reduction of the ratio, which attains its minimum values for $x_{F} \approx 0.15$; this corresponds to pions moving longitudinally at about the velocity of the spectator $\operatorname{system}\left(x_{F}=m_{\pi} / m_{p}=0.15, y=y_{\text {beam }}\right)$. This large distortion of the $\pi^{+} / \pi^{-}$ratio is a direct result of EM interaction between spectators and charged pions, as $\pi^{-}$are attracted and $\pi^{+}$repelled by the spectators.

A simplified Monte Carlo model that attempts to simulate the effect observed in $\mathrm{Pb}+\mathrm{Pb}$ $\left(\pi^{+} / \pi^{-}\right)$can be found in [11]. Spectators are modeled as stable, homogeneously charged spheres in their respective rest frames, and move away from the collision point at constant (beam) rapidity; final state pions are all produced at a single space-time point, and are propagated through the EM field generated by spectators via classical relativistic equations of motion. The model possesses 
one free parameter, the distance $d_{E}$ between the pion formation zone and the spectator system ( Fig.1(d)).

Fig.1(b) shows the results of the aforementioned Monte Carlo simulation for the particular value of $d_{E}=0.75 \mathrm{fm}$ that best reproduces the structure seen in NA49 $\mathrm{Pb}+\mathrm{Pb}$ data, Fig.1(a). In Fig.1(c), Monte Carlo simulation results for the $\pi^{+} / \pi^{-}$ratio are shown, for the full $x_{F}$ range, for a set of different values of $d_{E}$, namely $0 \mathrm{fm}, 0.5 \mathrm{fm}, 1 \mathrm{fm}$ and $1.5 \mathrm{fm}$. It is clear from Fig.1(c) that spectator-induced EM effects are highly sensitive to the distance $d_{E}$ between the pion formation zone and the spectators. Furthermore, by comparing Fig.1(c) to the experimental result in Fig.1(a), we can immediately exclude certain pion emission scenarios from the analysis. We therefore have evidence that spectator-induced EM effects can provide independent information about the spacetime evolution of the charged particle production process.
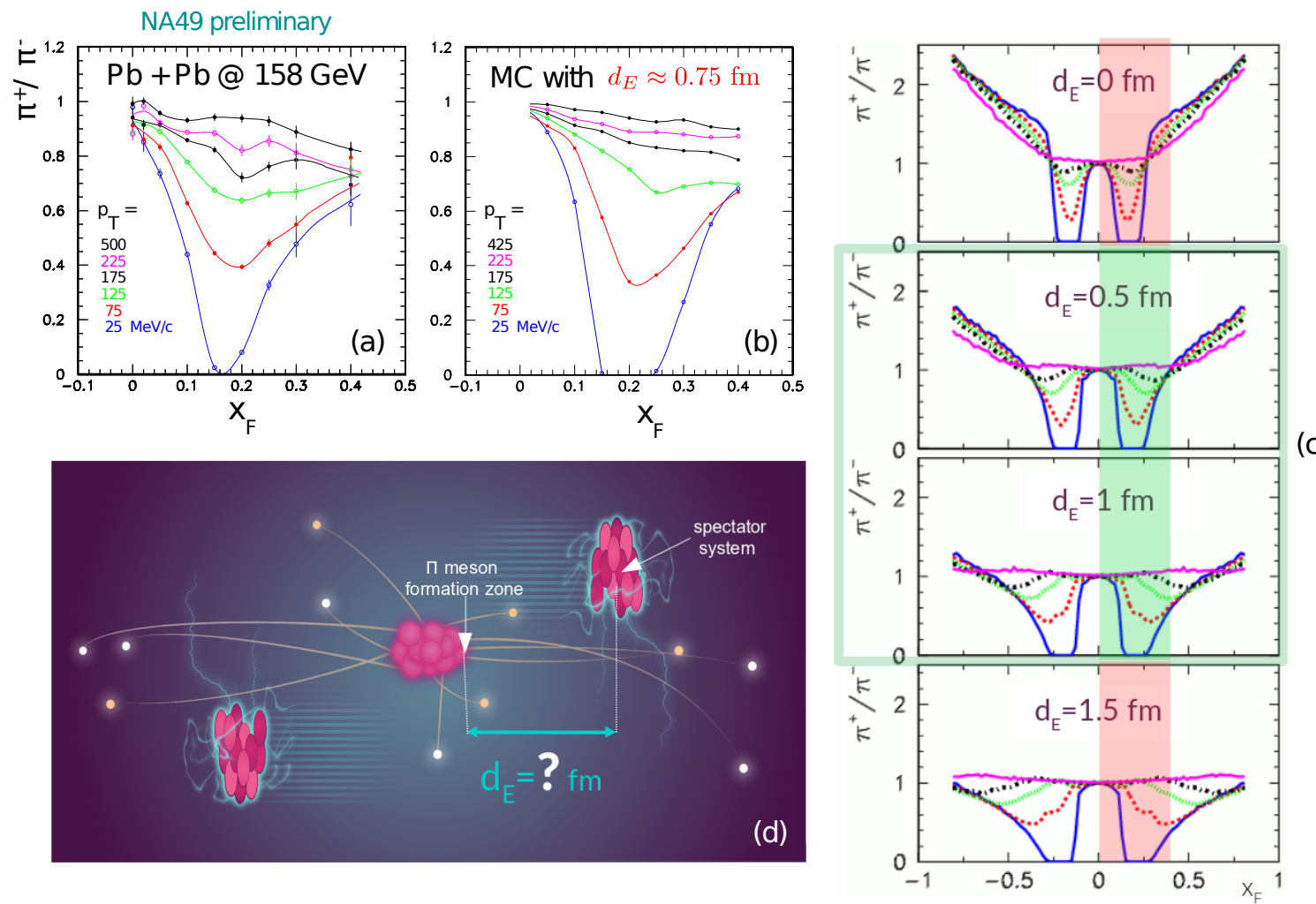

(c)

Figure 1: (a) Ratios of charged $\pi$ mesons $\left(\pi^{+} / \pi^{-}\right)$, drawn as a function of $x_{F}$ in nucleon-nucleon c.m.s. at several values of $p_{T}$, in NA49 peripheral $P b+P b$ reactions at $\sqrt{s_{N N}}=17.3 \mathrm{GeV}$ at CERN SPS [10] (b) Monte Carlo simulation of the same ratios for $d_{E}=0.75 \mathrm{fm}$ (c) Extended $x_{F}$ range Monte Carlo simulation results for the $\pi^{+} / \pi^{-}$ratio in peripheral $P b+P b$ collisions for selected values of $d_{E}$ [11]. The values of $d_{E}$ which match the experimental data are marked by a green frame. (d) Sketch of a non-central nucleusnucleus collision, illustrating the role of the adjustable $d_{E}$ parameter in the model simulation. [Image by I. Sputowska]

\subsection{EM effects on directed flow}

Other observables besides $\pi^{+} / \pi^{-}$ratio can furnish information about spectator induced EM effects as a function of pion rapidity. One such quantity is directed flow $v_{1}$ of pions, which is a 
measure of azimuthal anisotropy in pion production in nucleus-nucleus collisions (Fig.2). Fig.2(a) shows the directed flow of $\pi^{+} \& \pi^{-}$produced in $\mathrm{Au}+\mathrm{Au}$ collisions at $\sqrt{s_{N N}}=7.7 \mathrm{GeV}$ in the STAR experiment [2]. We observe a split in $v_{1}$ of oppositely charged particles. If we then apply our Monte Carlo simulation [12] and adjust the $d_{E}$ parameter, we obtain a fairly good agreement with STAR data points for $d_{E} \approx 3 \mathrm{fm}$, for pions moving at mid-rapidity.

Similarly, in Fig.2(b), we show the directed flow of $\pi^{+}$produced in $\mathrm{Pb}+\mathrm{Pb}$ collisions at $\sqrt{s_{N N}}=17.3 \mathrm{GeV}$ in the WA98 experiment [4]. Again, MC simulation provides a good description of the data for $d_{E} \approx 0.5 \mathrm{fm}$, for high-rapidity pions.

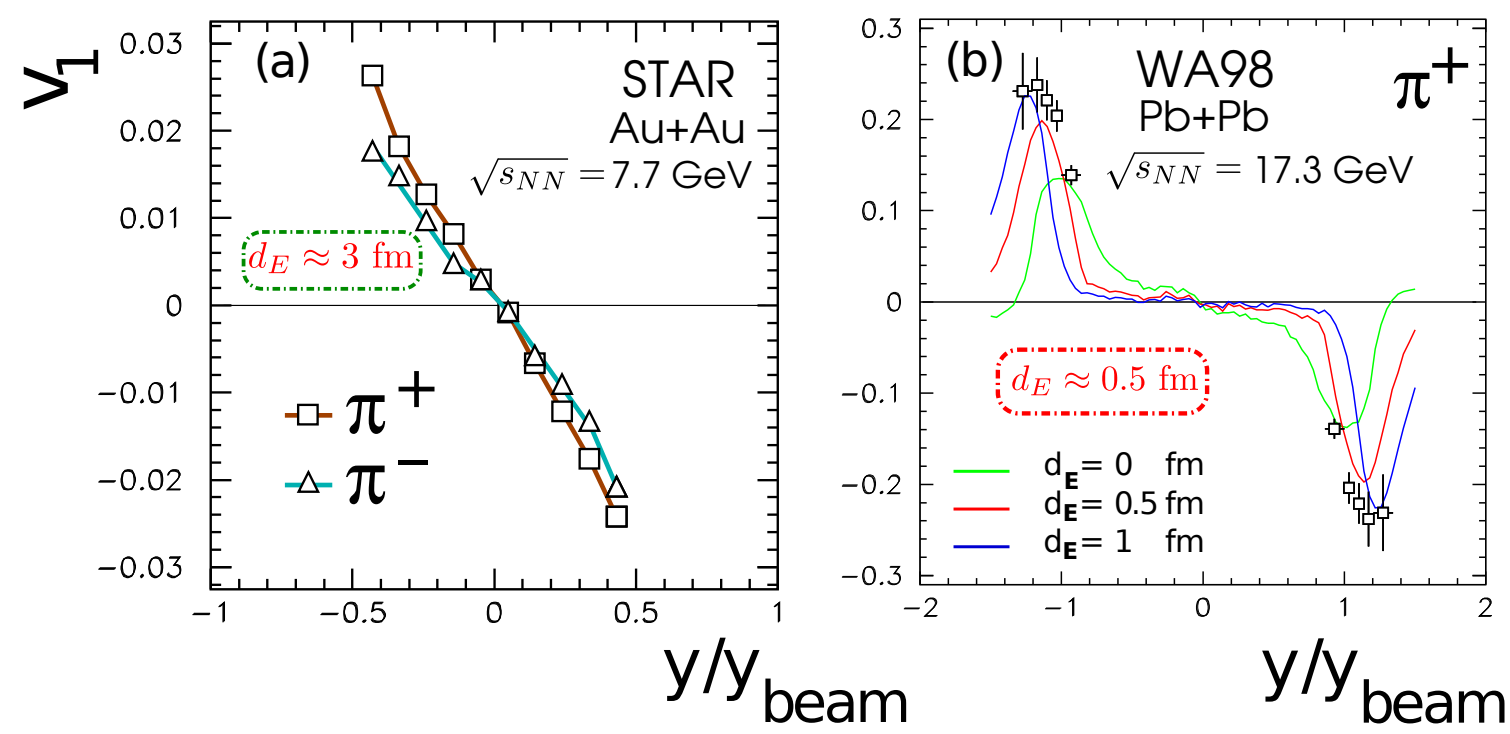

Figure 2: Directed flow $v_{1} \equiv\left\langle\cos \left(\phi-\Psi_{R P}\right)\right\rangle$ in a nucleus-nucleus collision for (a) $\pi^{+} \& \pi^{-}$produced in $\mathrm{Au}+\mathrm{Au}$ collisions at $\sqrt{s_{N N}}=7.7 \mathrm{GeV}$ in the STAR experiment [2]. The solid lines correspond to Monte Carlo simulation [12] for pions at mid-rapidity; (b) $\pi^{+}$produced in $\mathrm{Pb}+\mathrm{Pb}$ collisions at $\sqrt{s_{N N}}=17.3 \mathrm{GeV}$ in the WA98 experiment [4]. The solid lines correspond to Monte Carlo simulation [12] for high-rapidity pions.

\subsection{EM distortion of $\pi^{+} / \pi^{-}$ratio in NA61/SHINE Ar+Sc @ 150A GeV/c}

Fig. 3 shows a comparison of distortions in the $\pi^{+} / \pi^{-}$ratio, as a function of $x_{F}$ and $p_{T}$, in peripheral $\mathrm{Pb}+\mathrm{Pb}$ collisions at $158 \mathrm{~A} \mathrm{GeV} / \mathrm{c}$, presented in sec.2.1, with the same ratio calculated for intermediate and central $\mathrm{Ar}+\mathrm{Sc}$ collisions at $150 \mathrm{~A} \mathrm{GeV/c} \mathrm{[13].} \mathrm{This} \mathrm{is} \mathrm{the} \mathrm{first} \mathrm{observation} \mathrm{of}$ this kind of distortion in small systems at the SPS. While for peripheral $\mathrm{Pb}+\mathrm{Pb}$ (Fig.3(a); spectator charge $Q \approx 70$ ) the effect is quite large, in intermediate centrality $\operatorname{Ar}+\mathrm{Sc}$ (Fig.3(b); spectator charge $Q \approx 8$ ) it is still visible and large enough to break isospin symmetry. Interestingly, for the case of central $\mathrm{Ar}+\mathrm{Sc}$ (Fig.3(c)) a suppression of the ratio at beam rapidity may still be visible, suggesting a shadow of the EM distortion even for such a low spectator charge ( $Q \approx 3$ ).

An attempt to describe the pion ratio EM distortion effect on $\mathrm{Ar}+\mathrm{Sc}$ collisions through our stable spectator model is presented in Fig.4(a)-(b). Ar+Sc experimental data (black points) are compared to stable spectator Monte Carlo simulation (solid red lines) for $d_{E}=0.75 \mathrm{fm}$ and $d_{E}=$ $0.25 \mathrm{fm}$. The failure of the model to describe the data is evident, and it reveals that the stable 

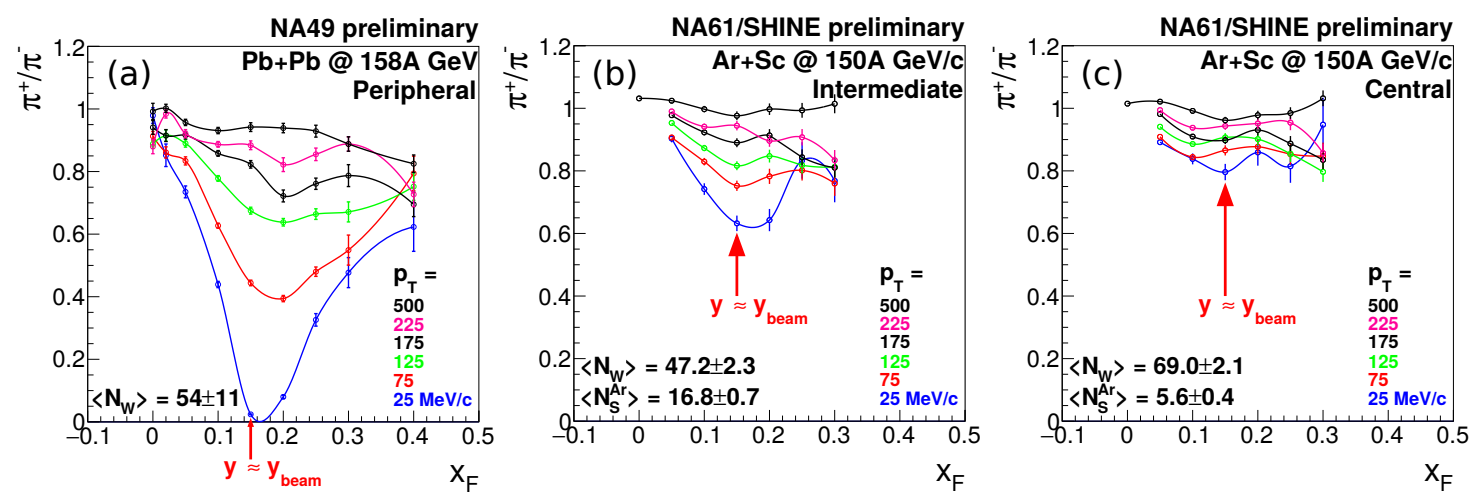

Figure 3: The $\pi^{+} / \pi^{-}$ratio as a function of $x_{F}$ for several values of transverse momentum in (a) peripheral $\mathrm{Pb}+\mathrm{Pb}$ [10], (b) intermediate centrality $\mathrm{Ar}+\mathrm{Sc}$, and (c) central Ar+Sc collisions [13] at the top CERN SPS beam momentum. Only statistical uncertainties are shown.

spectator approximation is not realistic. We therefore model spectator evolution, by introducing a radially (uniformly) expanding spectator, with surface velocity $\beta$, constant in its own frame. By adjusting this velocity $\beta$, we can achieve a good description of the experimentally observed ratio for $\beta \approx 0.4$ (dotted lines).

However, this poses a physical problem; the expansion velocity required to maintain agreement with data is not realistically attainable by the spectator system. It is therefore necessary to backtrack and re-examine our interpretation; instead of just the spectator charge, we must interpret the expanding charge distribution as the combined charge of both the spectators and the participants through which pions propagate. This opens the possibility that the center of momentum of the charge cloud moves at a different velocity than the spectator system. In fact, the optimal description, shown in Fig.4(c) by the solid yellow line, is obtained when we assume that the charge cloud moves slower than the spectator system. Our estimation then for the pion emission distance $d_{E}$ from the spectator system in the Ar+Sc system for $y \approx y_{\text {beam }}$ is $d_{E}=0.25 \pm 0.25 \mathrm{fm}$, with a deviation $\Delta y=-0.11$ for the expanding charge cloud w.r.t. spectator rapidity.

\subsection{Dependence of $d_{E}$ on pion rapidity}

Fig.5(left) summarizes the results obtained for $d_{E}$ by comparing our single point pion emission Monte Carlo model to experimental data shown in secs.2.1 - 2.3 (black data points) as a function of pion rapidity relative to beam rapidity, $y / y_{\text {beam }}$. A characteristic trend emerges, where pions moving at higher rapidities are produced closer to the spectator system.

In order to further investigate this result, a Monte-Carlo model was constructed [16], through which an intermediate system of various resonances with a proper lifetime $\tau$ is formed, which can be adjusted to fit the observed $d_{E}$ curve; subsequently, it decays into final-state pions. Results are illustrated by the red dots in Fig.5(left); as can be observed, the intermediate resonance model is in good agreement with the points obtained from the analyses described in secs. 2.1 - 2.3. 


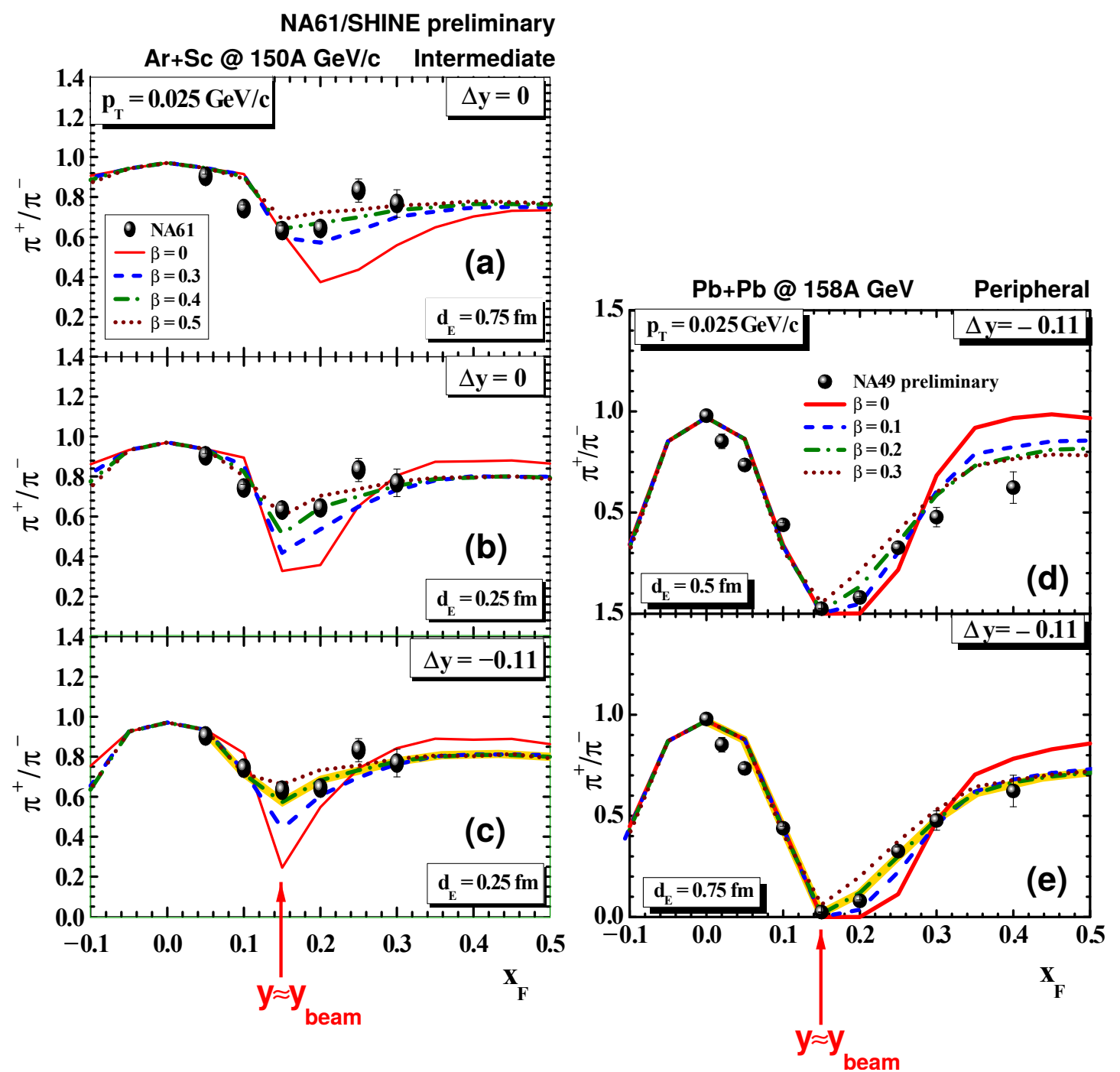

Figure 4: (a), (b), (c) Ratio of the number of emitted $\pi^{+}$over $\pi^{-}$measured in $A r+S c$ collisions at intermediate centrality by the NA61/SHINE Collaboration [1]. The experimental data points are compared to model simulations described in the text. The different combinations of $d_{E}, \beta$ and $\Delta y$ are indicated in the plots. The combination which we judge to give the optimal description of the data is indicated by the yellow line. (d), (e) Similar comparison as for the three previous panels, made for peripheral $\mathrm{Pb}+\mathrm{Pb}$ collisions measured by the NA49 experiment [14]. The optimal description of experimental data is indicated by the yellow line. The plots are redrawn from Refs. (a)-(c) [1], (d)-(e) [15].

\section{Longitudinal evolution of the system}

The observed trend of $d_{E}$ as a decreasing function of rapidity is an important piece of information gained from coupled studies of experimental data and Monte Carlo simulation. We therefore seek to decipher its message about the longitudinal evolution of the system.

In Ref. [7], a model for the evolution of heavy ion collisions is proposed, which has some similarity to the original "fire-streaks" model [17]. The nucleus-nucleus collision is pictured as the result of independent colliding "bricks" of nuclear matter, into which the nuclei are notionally 


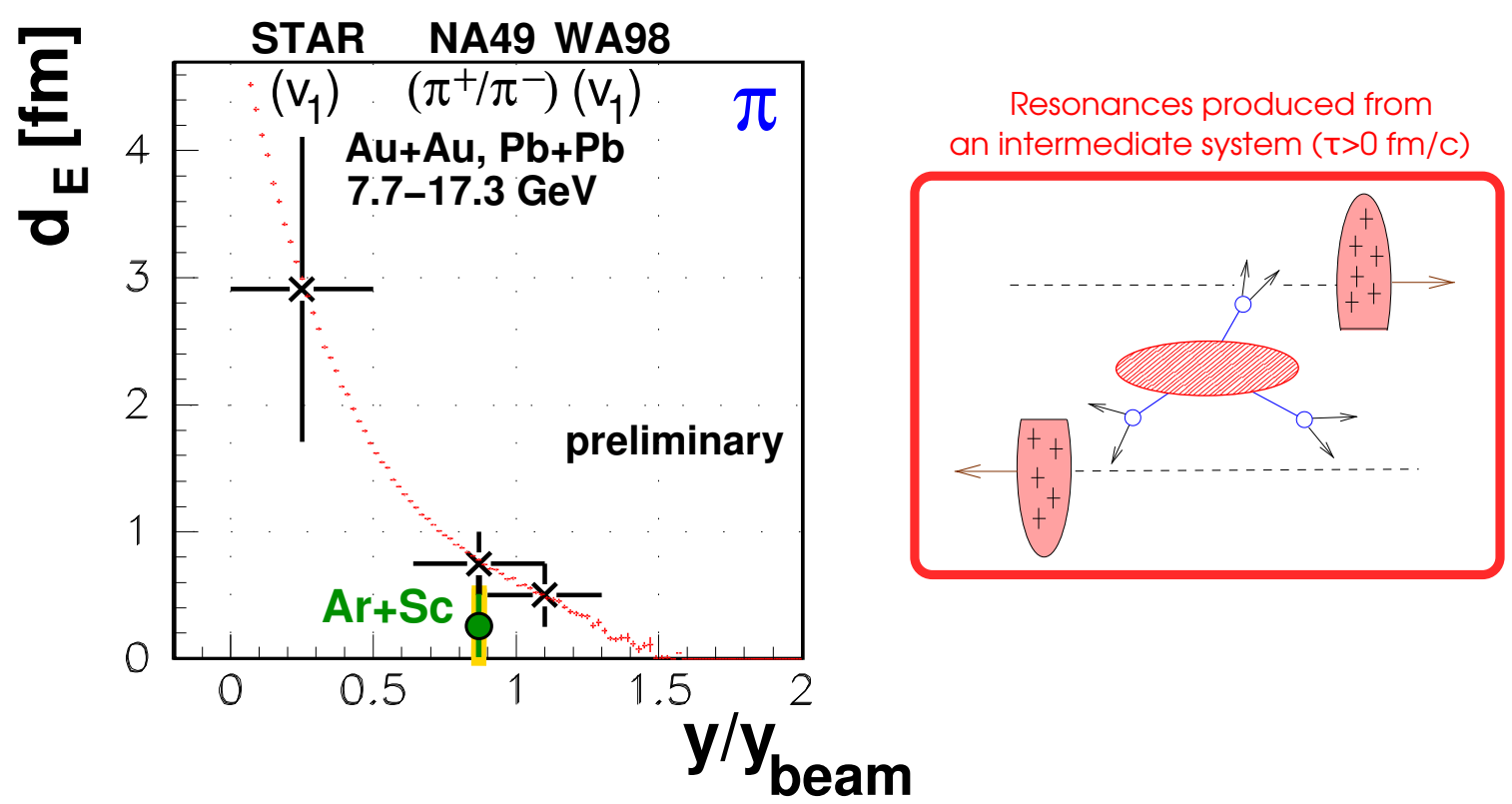

Figure 5: (left) Dependence of the pion emission distance $d_{E}$ on scaled pion rapidity (black data points), compared to the results of the Monte Carlo model simulation discussed in the text (red dots). (right) Resonance production model used to produce red dots line. Pions are produced from an intermediate system with a given proper lifetime $(\tau>0)$. Redrawn from [16].

partitioned in the direction transverse to the axis of collision (Fig.6(a)). The transverse size of the "bricks" is taken as $1 \times 1 \mathrm{fm}^{2}$. The resulting firestreaks of excited matter formed by the collisions (red) move according to local energy-momentum conservation, and independently fragment into new particles, whereas the two spectator systems (yellow) fly away from the collision with essentially unchanged velocities. In this model, the trajectories of pions originating from each firestreak will be affected by the EM fields induced by the spectator system; it is evident that the longitudinal distance $d_{E}$ between the emission zone of the pion and the spectator crucially depends on the rapidity $y_{s}$ of its originating firestreak.

We can model the independent fragmentation of each firestreak into pions [7], taking into account local energy-momentum conservation, as well as the available energy for the production of new particles:

$$
\frac{d n}{d y} \sim A \cdot\left(E_{s}^{*}-m_{s}\right) \cdot \exp \left(-\frac{\left[\left(y-y_{s}\right)^{2}+\varepsilon^{2}\right]^{r / 2}}{r \sigma_{y}^{r}}\right),
$$

where $E_{S}\left(m_{s}\right)$ is the energy (mass) of the firestreak, and $y_{s}$ its rapidity. This approach, based solely on local energy-momentum conservation, has remarkable predictive power. In Fig.6(b) the longitudinal spectra $d n / d y$ of $\pi^{-}$for NA49 $\mathrm{Pb}+\mathrm{Pb}$ collisions are shown [6], for central (red points) and peripheral (blue points) collisions, along with the firestreak model predictions (red/blue lines). We observe that the firestreak model can explain both the dependence of the yield and the broadening of the spectra with increasing peripherality of collision.

In Fig.6(c),(d), corresponding rapidity spectra are shown for $\pi^{-}$for NA61/SHINE Ar+Sc central collisions at $13 \mathrm{~A}-150 \mathrm{~A} \mathrm{GeV} / \mathrm{c}$ [18], as well as central and peripheral Be+Be collisions at 

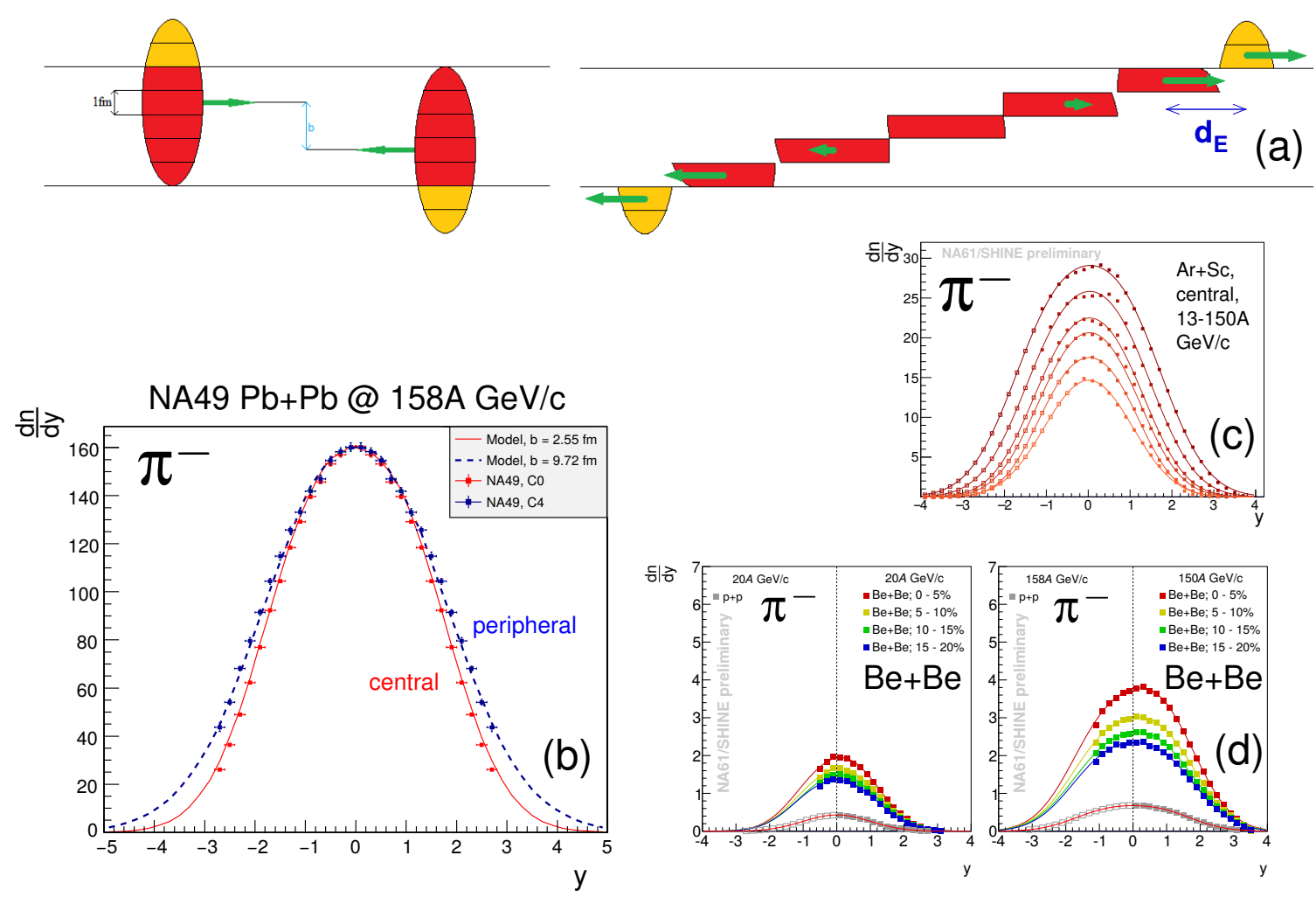

Figure 6: (a) Schematic sketch of the ultrarelativistic nucleus-nucleus collision made according to the model [7]. (b) Longitudinal spectra $d n / d y$ of $\pi^{-}$for $\mathrm{NA} 49 \mathrm{~Pb}+\mathrm{Pb}$ collisions at $158 \mathrm{~A} \mathrm{GeV} / \mathrm{c}$ [6], for central (red points) and peripheral (blue points) collisions; corresponding firestreak model predictions are shown (red/blue lines) (c) Longitudinal spectra $d n / d y$ of $\pi^{-}$for NA61/SHINE Ar+Sc central collisions at 13A$150 \mathrm{~A} \mathrm{GeV} / \mathrm{c}$ [18]. (d) Longitudinal spectra $d n / d y$ of $\pi^{-}$for NA61/SHINE Be+Be central \& peripheral collisions at 20A/150A GeV/c [19].

$20 \mathrm{~A} / 150 \mathrm{~A} \mathrm{GeV} / \mathrm{c}$ [19]. The extension of the aforementioned study to a variety of NA61/SHINE systems will be of great importance, given that NA61/SHINE already dedicates a lot of manpower \& work to performing a 2D scan in collision energy and system size, which could be augmented by a scan in the longitudinal evolution of the systems.

\section{Spectators}

It becomes clear from the discussion in sec. 3 that spectator-induced charge asymmetries in the longitudinal evolution of the distributions of charged particles produced in a heavy ion collision depend crucially on the space-time scale of the spectator break-up. Thus, we are prompted to study nuclear models of spectator de-excitation, usually studied for nuclear collisions at lower energies, in the context of ultrarelativistic heavy ion collisions.

Our analysis of the spectator system is focused on non-central $P b+P b$ collisions at $158 \mathrm{~A} \mathrm{GeV}$ beam energy. Spectator evolution is studied within a hybrid approach. First, the spectator excitation energy and the probabilities of its de-excitation processes are computed within the abrasionablation statistical model (ABRABLA) [20,21], applied with success to nuclear collisions at lower 


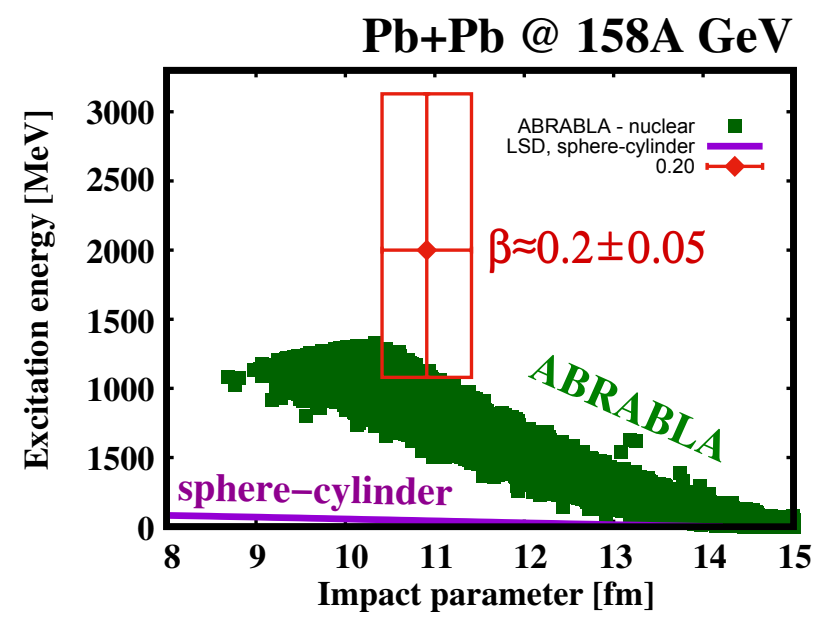

Figure 7: Excitation energy of the spectator in non-central ${ }^{208} \mathrm{~Pb}+{ }^{208} \mathrm{~Pb}$ collisions at $158 \mathrm{~A} \mathrm{GeV} \mathrm{beam}$ energy, computed in the abrasion-ablation model ABRABLA (green squares). The latter is compared to the excitation energy predicted by the geometrical model of sphere-cylinder collision (purple line). The red rectangle gives the estimated kinetic energy range for the expanding charged sphere described in the text. The plot is redrawn from [8].

energies; its application to ultrarelativistic $P b+P b$ reactions is described in Ref. [8]. Subsequently, for the ensemble of spectators obtained by the abrasion part of the ABRABLA code, their evolution in time is computed by solving transport equations of Langevin type. The details of the model can be found in Ref. [22].

In Fig. 7, we present the distribution of spectator excitation energies obtained in our ABRABLA sample of non-central $P b+P b$ collisions. As apparent from the plot, the range of impact parameters available in this sample is representative for the experimental sample of $P b+P b$ reactions from Fig. 4 (around $11 \mathrm{fm}$ on the average [23]). The ABRABLA calculation is compared to a geometrical estimate obtained by assuming a sphere-cylinder collision. The excitation energies obtained in the two approaches differ very significantly. For comparison, the figure includes also the estimate for the kinetic energy of a radially expanding charged sphere with the surface velocity $\beta=0.2 \pm 0.05$ which gives the optimal description of the EM effect on the $\pi^{+} / \pi^{-}$ratio (yellow line in Fig. 4(e)). It is apparent that the order of magnitude of this first estimate of kinetic energy of the charge cloud is rather comparable (within a factor of two with large uncertainties) to ABRABLA excitation energies at this impact parameter of the collision.

We therefore conclude that the study of EM effects may serve as an independent tool for providing information on the evolution of the spectator system, and possibly also differentiate between different models of the spectator initial stage. However, the role of participant net charge in the overall expansion of the effective charge cloud, section 2.3, also calls for clarification. In this respect, possible new measurements in the framework of the NA61/SHINE Phase II programme, recommended by the SPSC [9], could provide valuable information.

\section{Summary and conclusions}

This review aims to provide information on the multifaceted study of spectator-induced EM 
effects in ultrarelativistic heavy ion collisions, as presently pursued by the IFJ PAN group of the NA61/SHINE Collaboration. The first results on effects in charged pion emission are encouraging. Experimental results, combined with Monte Carlo simulations of EM effects, suggest a picture of the longitudinal evolution of the system at the initial stage at CERN SPS energies largely governed by energy-momentum conservation.

Additionally, EM effects provide a chance to get insight into the space-time evolution of the net positive charge cloud formed close to beam rapidity. Phenomenological studies of EM effects, put together with model calculations based on state-of-the-art nuclear theory, suggest that the magnitude of kinetic energy of expansion of the charge cloud made by the spectator and fast participant charge can be comparable, within a factor of two and with large uncertainties, to the excitation energy of the spectator system.

The present study can greatly benefit from an extension of the NA61/SHINE 2D scan (energy \& system size) in the direction of longitudinal evolution of the system, possibly with new measurements in the framework of the NA61/SHINE Phase II programme recommended by the SPSC.

Acknowledgments: This work was supported by the National Science Centre, Poland (grant no. 2014/14/E/ST2/00018).

\section{References}

[1] M. Kiełbowicz [NA61/SHINE Collaboration], to appear in Acta Phys. Polon. Supp. (2019).

[2] STAR Collab., L. Adamczyk et al., Phys. Rev. Lett. 112, 162301 (2014).

[3] A. Marcinek et al., Acta Phys. Polon. B49 711-718 (2018).

[4] WA98 Collab., H. Schlagheck, Nucl. Phys. A 663, 725 (2000).

[5] K. Aamodt et al., Phys. Lett. B 696, 328 (2011).

[6] T. Anticic et al., Phys. Rev. C 86, 054903 (2012).

[7] A. Szczurek, M. Kiełbowicz and A. Rybicki, Phys. Rev. C95, 024908 (2017).

[8] K. Mazurek et al., Phys. Rev. C97, 024604 (2018), and references therein.

[9] NA61/SHINE Collab., A. Aduszkiewicz et al., CERN-SPSC-2018-008.

[10] A. Rybicki, Acta Phys. Pol. B42, 867 (2011).

[11] A. Rybicki, A. Szczurek, Phys. Rev. C75, 054903 (2007).

[12] A. Rybicki et al., Acta Phys. Polon. B46, 737 (2015).

[13] A. Marcinek, EPJ Web Conf. 199, 02020 (2019).

[14] A. Rybicki [NA49 Collaboration], PoS EPS-HEP2009, 031 (2009).

[15] K. Mazurek et al., to appear in Acta Phys. Polon. Supp. (2019).

[16] A. Rybicki, A. Szczurek, M. Kiełbowicz, N. Davis and V. Ozvenchuk, Acta Phys. Polon. Supp. 9, 303 (2016).

[17] R. Hagedorn, Thermodynamics of Strong Interactions, CERN 71-12 (1971).

[18] M. Naskręt [NA61/SHINE Collaboration], PoS CPOD2017, 056 (2018). 
[19] E. Kaptur [NA61/SHINE Collaboration], PoS CPOD2014, 053 (2015).

[20] J.-J. Gaimard and K.-H. Schmidt, Nucl. Phys. A531, 709 (1991).

[21] A. Kelic, M.V. Ricciardi, and K.-H. Schmidt, arXiv:0906.4193v1, (2008).

[22] K. Mazurek et al., Eur. Phys. J. A53, 79 (2017).

[23] M. Kłusek-Gawenda, E. Kozik, A. Rybicki, I. Sputowska and A. Szczurek, Acta Phys. Polon. Supp. 6, 451 (2013), and references therein. 